\title{
ANÁLISIS DEL CONCEPTO 'EMPRENDEDOR' Y SU INCORPORACIÓN AL ÁMBITO EDUCATIVO
}

\author{
Analysis of the 'entrepreneur' concept and its incorporation \\ into education
}

Arantxa AZQueTa DíAz DE AlDÅ̊

Universidad Internacional de La Rioja. España. arantxa.azqueta@unir.net

bttps://orcid.org/0000-0003-2514-5989

Fecha de recepción: diciembre de 2018

Fecha de aceptación: febrero de 2019

\section{RESUMEN}

En la actualidad, en buena parte de los países desarrollados y en vías de desarrollo, se incentiva el impulso de la educación emprendedora por su contribución en la mejora socioeconómica. La inclusión de la competencia emprendedora como competencia básica despierta en los educadores e investigadores en educación el interés por conocer este fenómeno emergente que ha trascendido a la educación. Este artículo estudia el término emprendedor desde el ámbito semántico y conceptual. Plantea un doble objetivo. Por un lado, conocer el origen y evolución etimológica del término. Por otro, analizar la tradición intelectual y las principales teorías que recoge el concepto. Se sigue una metodología de análisis histórico-interpretativo con una revisión bibliográfica de los principales autores que han influido en su desarrollo. Se busca comprender el significado del término y los valores que encierra el concepto y contribuir al debate sobre su correcta aplicación en el ámbito educativo. El análisis ha permitido conocer el origen de los indicadores específicos de la competencia emprendedora junto a las influencias filosóficas que han asentado el emprendimiento. Se evidencia la fuerte identidad económica del concepto que deja oculto el sentido más extenso del término. Se concluye la necesidad de incidir en el significado originario que se relaciona con el desarrollo de la iniciativa y el desempeño de acciones que 
entrañan dificultad, y, revalorizar las notas de la competencia que hacen referencia a su dimensión personal y social. De esta manera, la educación emprendedora contribuirá a la forja de personalidades con iniciativa y capacidad de cooperación.

Palabras clave: emprendedor; educación emprendedora; antropología educativa; educación y desarrollo.

\section{ABSTRACT}

At present, many countries, whether developed or developing, are stimulating entrepreneurship education, due to its positive impact on socio-economic development. The inclusion of entrepreneurship as a basic competence raises interest, both among educators and researchers and beyond education. This paper analyses the term entrepreneur from a semantic and conceptual view. Its aims are, firstly, to show the origin and etymological evolution of the term, and, secondly, to analyze the intellectual tradition and the main theories converging in its significance. The methodology is an historical-interpretative analysis, and a bibliographic review of the authors that have influenced in the definition of the concept. The end prosecuted is to better understand the significance of the term and the values included in it, and also to make a contribution to the debate about its right application in educational environments. This analysis reveals the origin of some indicators related to the entrepreneurial competency, with the contribution of philosophical influences that have consolidated the entrepreneurship. It makes evidence of a strong economical identity that ignores the extended meaning of the term. The conclusion advocates for a recovery of the original sense of the term "entrepreneur». It is also required to give a new value to those notes of the competency referred to the personal and social dimension. In this way, the education for entrepreneurship will better contribute to the construction of personalities with initiative and ability to cooperate.

Key-words: entrepreneur; entrepreneurship education; educational anthropology; educational development.

\section{INTRODUCCIÓN}

Desde hace unos años, el fomento de una cultura emprendedora se considera un medio para favorecer el cambio de mentalidad que necesita la sociedad europea para adaptarse a los requerimientos del siglo XXI (Asamblea General, 2000; 2015; Consejo Europeo, 2000; Comisión Europea, 2014). Esta situación ha aumentado el interés por la educación emprendedora (Foss y Gibson, 2015; McClure, 2015; Nuñez Cubero, 2015; Nabi, Liñán, Fayolle, Krueger y Walmsley, 2017; Hornsby, Messersmith, Rutherford y Simmons, 2018).

En el ámbito español, tanto la LOE (2/2006) como la LOMCE (8/2013) señalan su compromiso con los objetivos planteados por Naciones Unidas y la Unión Europea desde el inicio del siglo XXI e incluyen la educación del talento emprendedor como 
uno de los objetivos de nuestro sistema educativo. A su vez, la Ley (14/2013), de apoyo a los emprendedores y su internacionalización, menciona la necesidad de reforzar la cultura emprendedora a través de la educación, para lo que solicita la inclusión del emprendimiento en el currículo.

El análisis de la bibliografía especializada ratifica que existe una abundante literatura sobre educación emprendedora. La voz entrepreneurship education arroja un total de 1.160.000 resultados en google académico. Igualmente, en la categoría education-educational research de la Web of Science para el periodo 1995-2017 se indexan 430 publicaciones científicas. Estos datos confirman el interés por esta disciplina desde muy variadas perspectivas, aunque prevalece el enfoque que lo asocia con el crecimiento económico.

La investigación académica es unánime al indicar, por un lado, la dificultad para definir la educación emprendedora (Hytti y O'Gorman, 2004; Pittaway y Cope, 2007; Mwasalwiba, 2010; Eurydice, 2016; Bacigalupo, Kampylis, Punie y Van den Brande, 2016; Lackéus, 2017; Bridge, 2017), y por otro, la ausencia de estudios de carácter teórico acerca de la misma (Tobón, 2001; Hannon, 2006; Gibb, 2011; Pepin, 2012; Lackéus, 2016; Bridge, 2017). Hayden (2012) analiza la producción científica de las principales revistas de filosofía de la educación de habla inglesa en el periodo 2000-2010 y concluye que entre la temática no se incluye ningún artículo ni se cita la educación emprendedora explícitamente. Para Lackéus (2016) esta ausencia de estudios que conjuguen estas dos disciplinas está motivada porque la teoría de la educación y el emprendimiento proceden de esferas de conocimiento dispares.

En el ámbito educativo en buena parte de los países desarrollados y en vías de desarrollo, se promueve el desarrollo e inclusión del emprendimiento, bien como asignatura independiente o bien como competencia transversal, en todas las etapas educativas, desde la educación temprana a la educación superior (Volkmann, Wilson, Mariotti, Rabuzzi, Vyakarnam, y Sepulveda, 2009; Mwasalwiba, 2010; Draycott y Rae, 2011; Rosendahl, Sloof y Van Praag, 2014; Moberg, 2014; Eurydice, 2016). Su aplicación práctica es heterogénea y prevalece un enfoque socioeconómico que se identifica con la mejora de las capacidades productivas y el desarrollo de las habilidades para generar empresas (Heinonen y Poikkijoki, 2006; Habiby, Deirdre y Coyle, 2010; Bernal-Guerrero y Cárdenas, 2017).

Este paradigma se basa en la premisa de que, en una economía madura, el crecimiento en cantidad y calidad de la actividad empresarial es proporcional a la calidad de la formación empresarial y se considera oportuno que se incorpore en todas las etapas educativas (Baumol, 1993; Nabi y Liñán, 2011). Sin embargo, esta opinión no es unánime y la educación para el emprendimiento no deja de estar exenta de controversias y opiniones dispares. Se reconoce su contribución positiva en la forja de las capacidades empresariales (Packham, Jones, Miller, Pickernell y Thomas, 2010; Westhead y Solesvik, 2015). Algunos autores se oponen con disparidad de argumentos: se plantean dudas sobre su eficacia (Matlay, 2006, 2007); se 
cuestiona el valor educativo que supone aprender a desarrollar un plan de negocio (Neck y Greene, 2011; Jones y Penaluna, 2013) o se considera que promueve la ideología de mercado y el neoliberalismo por lo que encuentra oposición en una parte del profesorado (Erkkilä, 2000; Johannisson, 2010; Komulainen, Naskali, Korhonen y Keskitalo-Foley, 2011; Berglund, 2013; Gill, 2014; Fougère, Segercrantz y Seeck, 2017; Lackéus, 2017). Algunos autores proponen que la educación para el emprendimiento en los ciclos de primaria apoye el desarrollo de las habilidades emprendedoras de los alumnos, pero con un enfoque no comercial (Iredale, 1993; Lewis y Massey, 2003; Hitty, 2008).

Los intereses políticos han convertido la educación para el emprendimiento en un objetivo educativo. No obstante, si esta se ciñe al desarrollo del tejido empresarial y productivo, cabe el riesgo de que se convierta en una pedagogía de la empresarialidad (Bernal-Guerrero, 2015). De este modo, si la educación temprana en emprendimiento carece de sentido educativo cabría cuestionarse la conveniencia de defender una formación temprana especializada en otras opciones profesionales o asociada a otras formas de vida (Barroso, 2015).

La inclusión de la competencia emprendedora como competencia básica imprime a los investigadores en educación la responsabilidad de aportar una fundamentación teórica que enriquezca el conocimiento acerca de la educación emprendedora. De acuerdo con Villamor y Prieto (2014) y Barroso (2015) es de interés abordar el análisis del término antes de introducirlo en el sistema educativo. En esta línea, este artículo fija la atención sobre el término emprendedor tanto desde el punto de vista semántico como conceptual. Busca conocer la génesis del concepto y su evolución y contribuir de esta manera a la reflexión y el debate y reforzar, en definitiva, la dimensión formativa de la educación para el emprendimiento y su implementación en las aulas.

\section{Metodología}

La lengua expresa el pensamiento y en el lenguaje influyen tanto el contexto como el marco de uso. Para Lakoff (1987) el lenguaje se basa en la experiencia y es un instrumento de clasificación que permite, a partir de nuestra cognición y conceptualización, categorizar el mundo. Con el paso del tiempo, las palabras adquieren acepciones diversas, se enriquecen con connotaciones fruto de la historia que han vivido y del carácter vivo del lenguaje. Por este motivo, precisar el origen y evolución del vocablo se plantea como tarea indispensable a la hora de profundizar en el estudio de un concepto. Para García-Aretio, Ruiz-Corbella y García-Blanco (2009) este análisis aporta calidad comunicativa y cientificidad. Por tanto, no es un ejercicio de erudición histórica, sino que se plantea un doble objetivo. Por un lado, conocer el origen y evolución etimológica del término. Por otro, analizarla tradición intelectual que recoge el concepto. Se busca, comprender tanto su significado como 
los valores que encierra de manera que el término se incorpore adecuadamente en el ámbito educativo.

La metodología de investigación de este trabajo es la propia de un análisis cualitativo que sigue un enfoque de estudio histórico-interpretativo. Se ha elegido este método porque se considera el apropiado para un trabajo de tipo conceptual y teórico. Se plantea un doble objetivo:

En primer lugar, se lleva a cabo una revisión semántica del término desde una perspectiva histórica (apartado 3) y la incorporación en el vocabulario económico (apartado 4 y 5). Se utilizan fuentes escritas, prioritariamente diccionarios de lengua, tanto históricos como etimológicos. Se considera que los diccionarios son una fuente relevante en el conocimiento de un término porque facilitan las claves para la comprensión de los textos escritos (RAE, 2001). A su vez son expresión de la madurez cultural de una comunidad lingüística y señalan la permeabilidad de la lengua a las cuestiones culturales y sociales del momento en el que se redacta (Ahumada, 2017). El Diccionario no recoge usos efímeros, sino que registra prácticas consolidadas en cada época histórica y como tal es reflejo de la ideología del momento.

En segundo lugar, se realiza una revisión conceptual para la que se han seleccionado autores que, desde su incorporación en el vocabulario económico en el siglo XVIII a nuestros días, han contribuido a la forja del concepto moderno de emprendedor (apartado 6, 7 y 8). Se ha procurado resaltar, siguiendo un orden cronológico, las principales teorías que influyen en la forja del concepto de manera que se comprendan las ideas y valores que le dan soporte y han conformado la manera de entender este fenómeno emergente. Se busca comprender la realidad y el fenómeno emprendedor y aportar así algunas ideas que faciliten su incorporación en la educación (apartado 9).

\section{ESTUDIO SEMÁNTICO DEL TÉRMINO EMPRENDEDOR: ORIGEN ETIMOLÓGICO, USO Y SIGNIFICADO}

Etimológicamente, los términos, emprendedor y emprender provienen del francés, entrepreneur y entreprendre respectivamente. A su vez, proceden de la misma raíz del latín vulgar (in, en, y prendĕre) o del latín clásico (apprehendĕre) cuyo significado es 'coger, atrapar, tomar' (Coromines, 1981). Esta raíz es común a las lenguas romance y explica términos como intraprendere en italiano, entreprendre en francés o prender/emprender en español. El Diccionario muy copioso de la lengua española y francesa equipara el verbo emprender español al entreprendre francés (Pallet, 1604).

En los siguientes apartados se recoge la evolución del término en dos idiomas: en español, ámbito en el que se encuadra la presente investigación, y en francés porque es la lengua que más ha influido en la forja del concepto de emprendedor. 


\subsection{En español}

El primer uso del verbo prender registrado en castellano corresponde a documentos aragoneses de 1030 y 1095 con el sentido de 'tomar' (Coromines, 1981).

En el siglo XIII, Gonzalo de Berceo introduce este término en dos pasajes con significados diferentes:

- En el primer caso como 'engendrar': "la cepa es buena, emprendió buen sarmiento" (Berceo, 1978, I, 9).

- En el segundo como 'tomar': "Sennor, bien te conseio que nada emprendas» (Berceo, 1978, I, 141).

El primer diccionario monolingüe del castellano que define el léxico castellano en castellano lleva por título Tesoro de la lengua castellana o española. Lo redacta Sebastián de Covarrubias (1611) y define el verbo 'emprender' como «determinarse a tratar algún negocio arduo y dificultoso. Se le pone aquel intento en la cabeza y procura executarlo» (p. 345). Este mismo significado perdura en el Diccionario de Autoridades (1726-1739) tanto para el verbo 'emprender' como para el sustantivo 'emprendedor'. Define el primero como: «determinarse a tratar y hacer alguna cosa árdua y dificultosa: como una facción militar, una conquista, etc. El que resuelve executar algún negocio y empressa de conseqüencia, antecedentemente la considera y dispone, para lograr su intento" (RAE, 1732, III). Define al emprendedor como «la persona que emprende y se determina a hacer y executar, con resolución y empéño, alguna operación considerable y árdua» (RAE, 1732, III). Esta definición perdura en la $1^{\mathrm{a}}$ ed. del Diccionario de la Real Academia de la Lengua (RAE, 1780).

Durante los siglos XVI-XVIII se da el nombre emprendedor a los que dirigen expediciones militares, capitanes de fortuna que reclutan tropas mercenarias para ponerse al servicio de príncipes, ligas o ciudades (Toro y Ortegón, 1999; PérezSánchez, 2011). Se resalta la característica propia del emprendedor que aborda una conquista con iniciativa y arrojo y se aplica a todo tipo de aventureros. Por asociación con el Nuevo Mundo este significado se hace extensivo a los llamados "maestro de obra" o "contratistas». Estas personas reciben, por encargo real, la producción de un bien. Esta actividad se desarrolla prioritariamente con las Indias Occidentales. Los contratistas reales financiaban sus actividades comerciales con la seguridad de que sus gastos estaban respaldados por los contratos previos (Verin, 2011). Por tanto, el término emprendedor reúne ambas apreciaciones, la referencia al Nuevo Mundo y la relación con los contratos de producción, pero carentes, en estos momentos, de riesgo.

Entre los diccionarios histórico-etimológicos resulta revelador el estudio de Cuervo (1998). Este diccionario es una fuente relevante porque señala el origen y la evolución semántica y formal de las palabras. Destaca tres significados del término emprender -en español-: 
1. Significado de 'acometer' con las siguientes variaciones:

a) 'Acometer' como verbo transitivo: supone lanzarse de manera resuelta y decidida e incluye implícitamente la idea de riesgo o arrojo.

b) 'Acometer' como verbo intransitivo: se refiere a dar comienzo, empezar o intentar.

c) Una tercera variación que recoge conjuntamente ambos sentidos como 'acometer' y como 'principiar'.

2. Significado de 'arrojarse' sobre alguna plaza u objetivo militar para atacarlo y para arrojarse con presteza sobre personas con intención de atacar. En ocasiones, el verbo significa entrar en una tierra o introducirse en el sitio atacado.

3. Y un tercer uso minoritario que se refiere a 'encender el fuego'.

El estudio de la definición de los términos 'emprender' y 'emprendedor' en el Diccionario de la Real Academia Española desde la $1^{\text {a }}$ edición de 1780 a la $23^{\text {a }}$ edición de 2014 también arroja luz acerca del proceso de creación de significado:

El sentido del verbo 'emprender' que se mantiene a lo largo del tiempo lo equipara con acciones que suponen 'comenzar' o 'tener iniciativa'. De manera implícita posee una cualidad que significa acometer con resolución, superar dificultades y tener capacidad de decisión. La $1^{a}$ edición identifica como actividades dificultosas las acciones militares o las conquistas (RAE, 1780). La definición se simplifica a partir de la $3^{\text {a }}$ edición que define 'emprender' como comenzar alguna cosa que encierra dificultad o peligro (RAE, 1791). El vínculo del verbo 'emprender' con una actividad de índole económica no se muestra hasta la $15^{\text {a }}$ edición "acometer e iniciar una obra, un negocio o un empeño que entrañan dificultad o peligro» (RAE, 1925). Este significado perdura actualmente (RAE, 2014).

Respecto al término 'emprendedor' se mantiene la definición que lo describe como "persona o entidad que emprende [acomete] con resolución acciones dificultosas o azarosas» (RAE, 2001). No se encuentran referencias al desempeño de actividades económicas. Únicamente, la $23^{a}$ edición matiza la definición anterior e incluye una referencia a la innovación y lo define como «que [el que] emprende con resolución acciones o empresas innovadoras» (RAE, 2014). Estas diferencias reflejan cómo el lenguaje cotidiano en español ha popularizado el término emprendedor y lo ha identificado con la generación de empresas, especialmente con empresas de alto rendimiento y naturaleza tecnológica, conocidas como start up.

Se observa un uso cada vez mayor del término emprendedor para referirse al emprendedor, figuras que con frecuencia se identifican en español. La explicación a esta cuestión es doble.

En primer lugar, se aprecia la influencia anglosajona (Veciana, 1996). El inglés no dispone de un significante propio y desde principios del siglo XVIII emplea el vocablo prestado francés entrepreneur (emprendedor) y el término entrepreneurship (emprendimiento) para referirse tanto al empresario como al emprendedor. Resulta 
complejo distinguir al entrepreneurship empresario del entrepreneurship emprendedor y solo el contexto los diferencia (Nueno, 2001; González-Domínguez, 2004).

En segundo lugar, el lenguaje cotidiano populariza el término 'emprendedor' y lo identifica con la generación de empresas. A partir de los años 50 del siglo XX se abandona el uso del vocablo 'empresario' y se sustituye por el de 'emprendedor'. Esto es así porque el término 'empresario' arrastra una percepción negativa puesto que se asocia con la disponibilidad de capital (Veciana, 2005). La expresión 'emprendedor' recoge el significado del entrepreneur francés y se acepta tanto en el lenguaje cotidiano como en el ámbito técnico-científico.

\subsection{En francés}

Las primeras referencias conocidas del término entreprendre corresponden a 1176 y significa 'comenzar' algo. Se confunde con la palabra enprendre que significa 'iniciar' o 'poner en práctica' (Ray, 1994, p. 700). Este significado es el que pervive principalmente a lo largo del tiempo como muestran los principales diccionarios franceses:

- En 1549 el erudito e impresor del rey Francisco I, Robert Estienne publica su Dictionarium latino-gallicum donde define, en latín, la palabra entreprendre como «aggredi aliquid, suscipere» que significa 'lograr algo, acometer' (Estienne, 1549).

- Esta definición pervive en diccionarios posteriores como son los de Thierry (1564) y Nicot (1606).

- Sin embargo, el primer diccionario en francés, homónimo del Covarrubias español, como es el Dictionnaire Français des Mots et des Choses de Pierre Richelet (1680) no recoge la expresión entreprendre porque solo incluye términos cultos.

- A comienzos del siglo XVIII, se amplía el significado en francés, tal y como recoge el Dictionnaire universel, contenant généralement tous les mots François, tant vieux que modernes \& les termes des sciences et des arts de Antoine de Furetière (1609-1688). Al igual que en español a partir del siglo XVIII se incluyen dos variantes (Furetière, 1708):

- En primer lugar, hace referencia al constructor de obras públicas que asume el encargo de construcción mediante contrato, "empresario" marítimo o que se dedica a abastecer al ejército de alimentos y municiones y trabaja con un precio establecido. Estas actividades requieren, no solo conocimiento técnico de las labores de construcción, sino que suponen una actividad comercial y económica.

- En segundo lugar, se describe al noble que explota las tierras de su propiedad para obtener beneficios con la venta de los productos (Hoselitz, 1951; Verin, 2011). 
A partir de este momento se produce un hito importante porque el término francés entrepreneur se incluye en el vocabulario económico.

\section{LA INCORPORACIÓN DEL TÉRMINO EMPRENDEDOR-ENTREPRENEUR EN EL DISCURSO ECONÓMICO}

El primero en introducir la voz entrepreneur en el lenguaje económico es Richard Cantillon (1680-1734). Su obra Essai sur la Nature du Commerce en Général (1730) ostenta el mérito de ser el primer escrito de la llamada «economía política» y Cantillon «el padre de la economía de empresa» (Aspromourgos, 1997; Seamus, 2013).

La época histórica de Cantillon se caracteriza por el crecimiento de las ciudades. La necesidad de adquirir provisiones que se producen fuera del perímetro de las ciudades permite desarrollar actividades comerciales. Se crean mercados que se convierten en la primera gran oportunidad para el entrepreneur. Este se transforma en un facilitador, en un intermediario entre el consumidor y los productores, incrementando al producto el valor añadido correspondiente a su traslado y comercialización.

La tarea emprendedora se convierte en un elemento dinamizador de la economía de las ciudades donde se establecen abundantes sinergias, pero también numerosos peligros que acechan al comerciante y que son fruto de los altibajos entre la oferta y la demanda. Los precios del mercado no los marca el valor intrínseco de los productos, sino que son resultado de las fluctuaciones entre la oferta y la demanda y pueden exponer al entrepreneur a situaciones de riesgo. La especulación, en cierta medida, entra a formar parte de la actividad empresarial.

En su trabajo, Cantillon reflexiona acerca del entrepreneur, define el perfil de la persona emprendedora y delimita las características del modelo económico que le asiste próximo al capitalismo en sus primeras etapas de desarrollo. Los principales rasgos que caracterizan al entrepreneur no son la disponibilidad monetaria ni la pertenencia a la clase propietaria, sino su capacidad para generar riqueza, producción y consumo y la asunción voluntaria de riesgos en condiciones de incertidumbre (Van Praag, 1999; Florea y Florea, 2013).

La incorporación por parte de Cantillon del concepto en el ámbito económico marca el concepto y su evolución posterior. El entrepreneur se convierte en un activo en la economía financiera «alguien que pone sus capacidades al servicio de la dinámica económica esperando, con ello, poder obtener el beneficio de la autosubsistencia o, en el mejor de los casos, el enriquecimiento (no sin riesgo de bancarrota)» (Barroso, 2015, p. 136).

El primer diccionario que refleja la vinculación del término entrepreneur a actividades económicas es el Dictionnaire universel du commerce de Savary (1724). Los hermanos Savary preparan, para uso personal, una lista alfabética de palabras relacionadas con el comercio y la industria, junto con ordenanzas e impuestos franceses o extranjeros. Explican quién es el entrepreneur, «aquel que emprende una obra. Se dice: un empresario de manufactura, un empresario de la construcción; un 
fabricante» (Savary, 1748, II, p. 1050). Posteriormente, los enciclopedistas Diderot y D'Alembert (1751) incluyen el término entrepreneur, en L'Encyclopédie ou Dictionnaire raisonné des sciences, des arts et des métiers que conserva las definiciones propuestas por los hermanos Savary. Definen el verbo entreprendre como cuidar por el éxito de un negocio, un oficio, una fábrica o un edificio (Éncyclopedie, 1751). Con respecto al entrepreneur destacan su actividad económica como productor de manufacturas o construcción, carente de riesgo (Éncyclopedie, 1751).

El término entrepreneur se divulga y se extiende también a Inglaterra donde sustituye a expresiones como merchant adventurer o undertaker que reducen su significado, ya que se refieren, respectivamente al comerciante y al empresario de pompas fúnebres. Sin embargo, no se generaliza el uso de la palabra entrepreneur en obras de economía hasta finales del siglo XVIII.

\section{EL EMPRENDEDOR A PARTIR DE LA REVOLUCión INDUSTRIAL DEL SIGLO XIX}

Si Cantillon introduce el término entrepreneur en el discurso económico en el siglo XVIII, la Revolución Industrial de mediados del XIX asimila al entrepreneur con el empresario. Desde una perspectiva histórica, la Revolución Industrial provoca que se identifique el crecimiento económico con la industrialización, síntoma de progreso. El grado de desarrollo de los países se mide en función de su nivel de industrialización y se considera que este es sinónimo de civilización y bienestar material (Martínez-Rodríguez y Amador, 2010).

En este contexto, el entrepreneur comienza a ajustarse a las nuevas demandas que exige el emergente desarrollo industrial y se torna en un empresario multifacético que asume las tareas de un gestor y amplía sus labores a otras actividades como la organización de la producción, la distribución del producto o la concentración de capital.

Entre los autores que contribuyen a asentar el significado moderno de emprendedor destaca Jean-Batipste Say (1767-1832) que define a entrepreneur como «el que está expuesto a todos los riesgos, pero en cambio se aprovecha de todo lo que puede serle favorable» (Say, 1821, pp. 413). Se dibujan con cierta claridad los rasgos que perduran en la definición del entrepreneur-empresario, hasta bien entrado el siglo XX: la asunción de riesgos en condiciones de incertidumbre a la que Say añade la capacidad de aprovechar las oportunidades. La experiencia profesional de Say al frente de un negocio industrial y los años de docencia en el Conservatoire des Arts et Métiers de París le llevan a conceder especial protagonismo a la formación de las capacidades empresariales, a las que concibe como fundamento del beneficio (Chassagne, 1983).

El concepto emprendedor recoge, principalmente en el ámbito galo, la influencia filosófica del utilitarismo (Mill, 1984). Esta teoría convierte la utilidad en modelo de vida y señala como criterio básico de la acción humana la búsqueda de beneficios y la maximización del bienestar. El utilitarismo económico transforma al 
empresario-emprendedor en una figura clave del proceso productivo porque facilita esta tarea y aporta valor al progreso económico y social. La utilidad reduce a un valor puramente instrumental donde el valor de las mercancías lo marcan las fluctuaciones de la oferta y la demanda que están influidas tanto por los costos de producción como por la utilidad.

\section{MARCO CONCEPTUAL QUE DA ORIGEN AL EMPRENDEDOR PROFESIONAL}

Los albores del siglo XX son una época histórica convulsa en la que la industrialización y la emergencia del capitalismo moderno irrumpen con fuerza en Occidente. A partir de este momento, el desarrollo del emprendedor corre paralelo a la institucionalización de la práctica económica capitalista y empresarial. La actividad emprendedora se convierte en un acto estrictamente económico y el emprendedor en un agente económico que dedica todo su empeño a la satisfacción de necesidades que se expresan en términos monetarios.

El continuador directo de la noción de emprendedor es Alfred Marshall (18421924), representante de la escuela Neoclásica, e impulsor de la «economía del bienestar», base del capitalismo económico. Marshall (1961) parte de los principios utilitaristas y rompe con la concepción de la teoría clásica del valor-trabajo, por el que los bienes tienen una valoración objetiva en función del trabajo, es decir, la fuerza de los hombres que han interactuado en el proceso de producción de dicho bien, junto a los costes de producción. Aplica el principio de utilidad marginal, por el que el precio de un bien no se define por la utilidad objetiva sino la utilidad marginal, en la que la escasez condiciona la utilidad y supone el aumento del precio. Este planteamiento desencadena la imposición progresiva de la soberanía de los consumidores porque los objetos dejan de tener un valor en sí, para depender de la utilidad que proporcionan al consumidor (Zaratiegui, 2002).

Este planteamiento, que expresa los valores de la Modernidad, hay que encontrarlas en el racionalismo, el subjetivismo y el individualismo metodológico, herederos del utilitarismo. Estas teorías contribuyen a asentar el capitalismo, que es una forma de racionalización, absolutizando al homo oeconomicus que aplica las categorías analíticas de raíz económica a todos los ámbitos. A su vez constituyen las bases ideológicas del pragmatismo y relativismo que con posterioridad forjarán el modelo social de la postmodernidad.

La aportación filosófica fundamental de este momento corresponde a los pensadores centroeuropeos -Carl Menger (1840-1921) y Max Weber (1864-1920)- que elaboran una teoría económica que influye en la conceptualización del emprendedor y que culmina en la profesionalización de esta actividad.

El eje de la reflexión lo constituye la teoría subjetiva del valor que sostiene que un bien adquiere valor en la medida que satisface una necesidad y se transforma en un bien económico. El valor de bien brota de su carácter económico, es decir, de la relación entre necesidad y bien y de la utilidad que proporciona al consumirse. Los 
bienes no económicos no tienen valor ni de cambio ni de uso, aunque sí pueden brindar utilidad. El valor de bien no está en las cosas sino en los individuos y depende de la significación que el individuo otorgue a las necesidades (Menger, 1997). Con este punto de partida, Menger considera al emprendedor en una figura relevante dentro de la economía porque se convierte en un sujeto productor de valor (Rodríguez Sedano, 1999).

La emergencia del emprendedor tiene su razón de ser en el intercambio y en un hecho central, la satisfacción de necesidades. Su teoría tiene varios pasos metodológicos: teoría del bien, teoría del valor y teoría del intercambio que se profesionaliza. La transformación de unos bienes de orden superior en otros de orden inferior requiere de alguien que haga los cálculos y encamine la actividad empresarial. El empresario-emprendedor asume esta tarea y su principal ocupación es prever, planificar, plantear las necesidades de producción y desarrollar intercambios que sean rentables y necesarios para la sociedad. A su vez, el valor final de los bienes depende de si satisface las necesidades y de la demanda que hagan los seres humanos de dichos bienes. Estas ideas posibilitan el desarrollo del empresarioemprendedor como un profesional que personaliza el espíritu burgués y encarna la ética calvinista (Weber, 1995).

Esta concepción supone la quiebra con la tradición escolástica en la que el fundamento del valor de bien responde a la verdad sobre el bien mismo. La razón define lo que es un bien objetivo que se constituye en objeto de la voluntad, determina en qué medida los bienes son acordes a la dignidad de la naturaleza humana y establece una jerarquía entre ellos. Sin embargo, según el planteamiento subjetivista, el valor de bien lo marcan de manera subjetiva los sentimientos o una razón autónoma del bien. Este planteamiento sitúa a la persona humana en un plano intramundano, olvida la orientación transcendente, limita la libertad personal a la satisfacción de necesidades, erosiona el fundamento del valor de bien y reduce la posibilidad de contemplar la propia existencia como don abierto a los demás. Progresivamente la actividad empresarial se constituye en vehículo de mejora social, se erige en responsable del progreso y se asientan las bases que configuran el nuevo paradigma emprendedor.

\section{EL EMPRENDEDOR INNOVADOR DE SCHUMPETER}

La revisión del pensamiento y tradición intelectual del concepto de emprendedor tiene en la figura de Joseph Schumpeter (1883-1950) una aportación importante. Schumpeter califica al empresario-emprendedor como un tipo excepcional, dotado de "espíritu emprendedor» (Schumpeter, 1966). Lo define como una persona intuitiva, con voluntad de cambio y transformación, rompe rutinas, tolera la incertidumbre, atrae la actividad económica y destaca por su tenacidad. El carácter innovador es su característica principal que ha permitido el desarrollo del capitalismo: consigue beneficios por su capacidad de innovación, abre posibilidades de mejora al añadir 
valor a los productos y permitir el aumento de precios. La capacidad de innovación se convierte en elemento definidor y particularidad de la persona emprendedora.

Para Rodríguez-Sedano (1999) otra característica del empresario-emprendedor es su sentido proyectivo de la realidad entendido como capacidad eficaz y decidida de construir el futuro. El carácter evolutivo del capitalismo y su tendencia al crecimiento sintonizan con el perfil transformador y dinamizador del emprendedor que cambia unos bienes de orden superior en otros de orden inferior.

Finalmente, un último aspecto a reseñar es la cuantificación monetaria que se convierte en el método de ponderar el valor porque es también un modo de racionalidad. Aunque en un principio esta se limita al orden económico, posteriormente se extiende a todos los sectores de la actividad humana. Este planteamiento convierte lo económico en el principal valor que marca el mercado y la acumulación de capital en el valor supremo porque permite comprar todo en una sociedad mercantilizada (Castells, 2017).

La tarea emprendedora se constituye en una actividad de orden superior que se profesionaliza (Weber, 1995) y que convierte a los emprendedores en pilares del crecimiento (Rodríguez-Sedano y López de Pedro, 2013; Baumol, 2015).

\section{EL NUEVO PARADIGMA EMPRENDEDOR: EL EMPRENDEDOR PILAR DEL CRECIMIENTO}

La influencia de Schumpeter en el desarrollo del concepto de emprendedor es innegable y sus aportaciones alimentan los enfoques del modelo empresarial de Frank Knight (1885-1972). Este introduce en el centro de la tarea emprendedora un nuevo elemento, la toma de decisiones como medio para reducir la incertidumbre y aprovechar las oportunidades atractivas (Knight, 2006).

Junto al emprendedor innovador de Schumperter y el emprendedor reductor de incertidumbre de Knight, resalta la contribución de la Nueva Escuela Austriaca que asume entre sus presupuestos la teoría subjetiva del valor y el individualismo metodológico. Esta corriente aglutina a economistas que propugnan una economía de intención pragmática que percibe oportunidades, tiene habilidad para aprovecharlas, toma decisiones para desarrollarlas y compite con otros empresarios que esperan entrar en los roles de la economía de mercado y conseguir beneficios (Van Praag, 1999; Huerta, 2002). Estos planteamientos llevan a que se asocie el fenómeno empresarial con el concepto de oportunidad para la generación de nuevas empresas que llevará a las instituciones públicas a impulsar proyectos de desarrollo empresarial partiendo del diagnóstico de oportunidades. Asimismo, ha permitido caracterizar el emprendimiento como un proceso que supone el descubrimiento, la evaluación y la explotación de oportunidades (Shane y Venkataraman, 2000). Este prisma aproxima el enfoque emprendedor a otros ámbitos de acción humana como son el cultural o el social porque se genera no solo valor económico sino también nuevos productos, servicios, procesos, acciones, etc.Esta perspectiva fortalece el interés educativo del enfoque emprendedor. 
El apoyo de instancias políticas y académicas asienta la reputación del emprendimiento que adquiere popularidad, un perfil de desempeño más alto y mayor relevancia (Laukkanen, 2000). Se debe a varias causas: la extensión del número de iniciativas empresariales, el cambio en la percepción social acerca del empresario, el aumento del prestigio del emprendimiento como actividad profesional y, finalmente, los avances tecnológicos que posibilitan la creación de nuevos productos comerciales y nuevas oportunidades empresariales (Vesper y Gartner, 1997). A partir de los años 80 del siglo XX el emprendimiento se convierte en un campo de estudio propio y reconocido por el mundo científico (Baumol, 1993; Cassis y Pepelasis, 2005). Esta situación favorece el interés por introducirlo en los sistemas educativos como materia que puede ser enseñada y convierte a las instituciones educativas en despertadores de la conciencia emprendedora.

Grosso modo se diferencian dos grandes grupos de educación emprendedora que dan respuesta heterogénea a las necesidades de formación: el «modelo americano» y el «modelo europeo» (Erkkilä, 2000; Hytti y O'Gorman, 2004; Gibb, 2006; Peña-Calvo, Cárdenas, Rodríguez-Martín y Sánchez-Lissen, 2015). La diferencia entre ambos modelos responde a que el americano se orienta directamente a la generación de empresas, mientras que el europeo sigue un modelo transversal cuyo objetivo es el desarrollo de personas emprendedoras a través de las competencias de creatividad, innovación e iniciativa personal que capaciten para, si fuere el caso y entre otras iniciativas posibles, crear una empresa.

\section{Algunas CONCluSiones Y SUGERENCIAS PARA SU INCluSión EN EL ÁMBito EDUCATIVO}

El análisis precedente ha puesto en evidencia algunas cuestiones.

En primer lugar, conviene reseñar cómo la ciencia económica ha sabido resaltar el concepto de emprendedor y ha destacado su valor para movilizar recursos, desarrollar la iniciativa, generar valor y crear riqueza (Brunet y Alarcón, 2004; Cassis y Pepelasis, 2005; Baumol, 2014). Estos aspectos, que tienen su raíz en el ámbito productivo y económico, se han implementado en el ámbito educativo y resultan indispensables en la aplicación educativa. Se ha generado un enfoque educativo específico que favorece el crecimiento del potencial emprendedor, contribuye al crecimiento integral y se proyecta en todas las esferas de la vida de las personas y no solo en la socieconómica.

El lenguaje coloquial y, sobre todo, la fuerza imparable de la economía desde el siglo XIX, han arrastrado al concepto de emprendedor, a tomar una óptica eminentemente económica que ha limitado su significado a este ámbito (Toro y Ortegón, 1999; Toca, 2010; Pfeilstetter, 2011; Bernal-Guerrero, 2014; Villamor y Prieto, 2014; García del Dujo, 2015). Aunque en los diccionarios se mantiene el significado original que lo relaciona con la iniciativa, el lenguaje habitual ha quedado oculto el significado más extenso que lo identifica con el comienzo de acciones que encierran dificultad. 
Un segundo aspecto, destaca la importancia que se concede al sujeto económico, como principal actor del desarrollo (Nueno, 2001; Veciana, 2005; Marulanda, Montoya y Vélez, 2014). Considerar el emprendimiento como fenómeno económico ha llevado a su revalorización y le ha agregado cierto carácter novedoso (García del Dujo, 2015). Sin embargo, aunque se trata de un fenómeno socialmente reciente, no es una idea nueva porque responde a una actitud antigua y a un quehacer específico del ser humano. El trabajo es natural y propio del ser hombre. Este no se limita a cubrir ciertas necesidades, no se conforma, sino que busca más, anhela más, trabaja para ir más allá y vencer dificultades porque es un ser capaz de metas y fines. Si es un "ser en el mundo» ${ }^{1}$ lo es, no solo para alimentarse, sino para asumirlo y transformarlo. Por esto el ser humano emprende, y esta tarea es propia de su naturaleza humana. Mientras que el animal no emprende; el hombre sí lo hace, porque tiene capacidad de plantearse metas y elegir con libertad los medios para alcanzar un fin (Segura, 1994).

Este sentido originario imprime al emprendimiento su dimensión educativa. La sociedad necesita crecer no solo económicamente sino también mejorar humanizándose. A esta tarea contribuye la educación emprendedora. El sentido perfectivo del ser humano le lleva a emprender, progresar, al aumento intensivo y extensivo de lo que posee, a buscar riqueza y bienestar material. También busca adquirir conocimiento y mejorar en su desarrollo ético: «de estos tres elementos, conocimiento, ética y riqueza, consta el vivir bien que es propio del ser humano en cuanto que le corresponde vivir como ser libre» (Altarejos, Rodríguez-Sedano y Fontrodona, 2003, p. 11). De esta manera, el emprendedor es un humanizador del entorno, un innovador, un facilitador de cambios. Alguien que crea algo diferente y con valor más allá de lo económico, capaz de beneficiar en primer lugar a la persona y en consecuencia a la sociedad. A su vez el emprendimiento es una forma de pensar, razonar y actuar que busca dar respuesta a las necesidades, destaca oportunidades, calcula el riesgo, se adapta al cambio y a la multidisplinariedad, se hace cargo de las situaciones con visión global. Por eso conviene que esté presente en todas las profesiones, no solo en las ciencias empresariales y que al mismo tiempo ejerza un liderazgo ético.

En tercer lugar, la revisión conceptual de autores que han contribuido a la forja del concepto moderno de emprendimiento ha permitido aclarar el origen de algunos de los indicadores más relevantes que se han asociado a la competencia emprendedora a lo largo del tiempo y que, a modo de síntesis, se desgranan en la siguiente tabla.

1. Esta expresión tiene su origen en Heidegger (1889-1976) y en su obra Ser y tiempo (1927). Describe al ser humano como Dasein, como existir y ser ahí. En consecuencia, adquiere importancia el mundo y la relación del ser humano con el mundo. 
Tabla 1. Revisión histórica de los indicadores asociados a la competencia emprendedora

\begin{tabular}{|l|l|}
\hline \multicolumn{1}{|c|}{ Indicador de la competencia } & \multicolumn{1}{c|}{ Contribución } \\
\hline $\begin{array}{l}\text { Desarrollo de la iniciativa } \\
\text { Disposición al logro }\end{array}$ & Siglos XV-XVI \\
\hline Asunción de riesgos & Cantillon \\
\hline Búsqueda de oportunidades & Say \\
\hline $\begin{array}{l}\text { Imaginación } \\
\text { Creatividad } \\
\text { Innovación } \\
\text { Liderazgo }\end{array}$ & Schumpeter \\
\hline Toma de decisiones & Knight \\
\hline
\end{tabular}

El análisis muestra cómo el concepto emprendedor recoge el aporte teórico del racionalismo, el subjetivismo y el individualismo metodológico. Estas tres teorías han contribuido a asentar el capitalismo, absolutizando al bomo oeconomicus. Este se caracteriza porque aplica categorías de raíz económica a todos los ámbitos, convierte el mercado y la satisfacción de necesidades en una prioridad y se desinteresa por la acción social, porque prima el individuo frente a la dimensión social y la responsabilidad compartida. Progresivamente, el término se ha ido cargando de economicismo. Se ha convertido en una categoría social, una forma de vida, un modelo cultural, un modo de estar y de relacionarse con el mundo que se profesionaliza y asume los principios del neoliberalismo (Pfeilstetter, 2011; Marttila, 2012; Kenny y Scriver, 2012; O' Rourke, 2014). Estos planteamientos transcienden al ámbito educativo que se ve urgido por la necesidad de hacer crecer la competitividad del tejido industrial (Bernal-Guerrero, 2015). Asimismo, García del Dujo (2011) señala que bajo los postulados utilitaristas se impone una pedagogización de la vida que interpreta la educación como herramienta de la sociedad para la transmisión de valores útiles. La educación se convierte en un instrumento para alcanzar un fin en el que se prioriza el utilitarismo y el racionalismo instrumental. En consecuencia, se concluye que esta perspectiva incentiva desarrollar la competencia emprendedora porque facilita el desarrollo tecnológico y la modernización de la sociedad y contribuye a la sostenibilidad de un modelo social preocupado mayoritariamente por el logro de beneficios económicos.

Este es el sustrato cultural que subyace a la cultura empresarial que ha convertido el individualismo en una forma de vida, y donde la obtención de beneficios a corto plazo es el principio rector de la toma de decisiones cada vez más arriesgadas. Posteriormente, el espíritu emprendedor allana el camino para la liberalización de la economía mundial y la globalización del libre mercado. Este bagaje ha contribuido a forjar un fenómeno de fuerte identidad económica, centrado en la generación valor económico. 
Finalmente, la inclusión del emprendimiento en las aulas requiere recuperar el significado originario del término emprendedor que se relaciona con el desarrollo de la iniciativa y el desempeño de acciones que entrañan dificultad. De esta manera la educación emprendedora es de interés en el ámbito educativo porque da respuesta a la aspiración natural de la persona al crecimiento y favorece el desarrollo y la mejora tanto de la persona como de la sociedad. En esta línea se incide en el desarrollo de los indicadores de la competencia que hacen referencia a la definición primera del emprendedor y en los que prima la dimensión personal y social frente al carácter económico. Estos indicadores aluden al cultivo de la iniciativa, el desarrollo de la autonomía, el tesón y la solución de problemas, la capacidad crítica, el valor de servicio, la solidaridad, la cooperación social y el pensamiento ético y sostenible. De esta manera se prioriza a la persona y se colabora en el crecimiento de todas las dimensiones del ser personal. Si la educación no tiene en cuenta las necesidades netamente personales limita el carácter formativo a lo puramente instrumental. En consecuencia, se devalúa el valor de la educación como medio para promover la realización personal. No se puede olvidar que la dimensión personal dilata la perspectiva conceptual. Ampliar la significación del emprendimiento se presenta como tarea imprescindible para hacer frente a los desafíos educativos de las próximas décadas y para su inclusión en las aulas. Se requiere una visión holística, integradora, multidimensional y transversal del emprendimiento que evite el reduccionismo economicista (Du Gay, 2004; Kenny y Scriver, 2012; O’ Rourke, 2014).

La educación es una tarea dispositiva, cuya primera acción es seleccionar lo que se enseña, de manera que los criterios de elección determinen que la enseñanza sea educativa y promueva la formación humana. Tanto la política y la economía como el sistema educativo nos impulsan hacia el hacer, la eficacia y la eficiencia del mercado. La educación emprendedora no es solo medio para producir, ni tampoco se puede reducir a un adiestramiento. Se requiere que esta se configure como materia que favorezca el crecimiento personal y posibilite una formación integral. El ser humano es capaz de crecer y la educación tiene como tarea ayudar al crecimiento humano (Polo, 1990). La educación no solo se adapta al medio o a las necesidades del momento, sino que contribuye al desarrollo e implica a la persona en la mejora social (Altarejos, 2006).

El desarrollo de la competencia emprendedora precisa recuperar el significado originario del término emprendedor y revalorizar la dimensión personal de manera que la educación emprendedora contribuya a la forja de personalidades con iniciativa y capacidad de cooperación.

\section{REFERENCIAS BIBLIOGRÁFICAS}

Ahumada, I. (2017). Diccionario bibliográfico de la metalexicografía del español. Jaén: Universidad de Jaén. 
Altarejos, F., Rodríguez-Sedano, A, y Fontrodona, J. (2003). Retos educativos de la globalización. Hacia una sociedad solidaria. Pamplona: Universidad de Navarra.

Altarejos, F. (2006). Estudio introductorio. Leonardo Polo: Pensar la educación. En L. Polo Ayudar a crecer, Cuestiones filosóficas de la educación (pp. 13-39). Pamplona: Universidad de Navarra.

Asamblea General (2000). Resolución 55/2. Declaración del Milenio (13 de septiembre de 2000), vol. 24. Recuperado de: http://www.un.org/spanish/milenio/ares552.pdf (Consultado el 12/12/2018)

Asamblea General (2015). Resolución 70/1. Transformar nuestro mundo: la Agenda2030 para el Desarrollo Sostenible. Septuagésimo periodo de sesiones. Temas 15 y 116 del programa (21 de octubre de 2015). Recuperado de: https://unctad.org/meetings/es/ SessionalDocuments/ares70d1_es.pdf (Consultado el 12/12/2018)

Aspromourgos, T. (1997). Cantillon on real wages and employment: a rational reconstruction of the significance of land utilization. Journal of the History of Economic Thought, 4(3), 417-443. https://doi.org/10.1080/10427719700000060

Bacigalupo, M., Kampylis, P., Punie, Y. y Van den Brande, G. (2016). EntreComp: The Entrepreneurship Competence Framework. Luxembourg: Publication Office of the European Union.

Barroso, C. (2015). Emprendedor: cuando el término es más que una palabra. En L. Núñez Cubero (Coord.), Cultura emprendedora y Educación (pp. 133-142). Sevilla: Universidad de Sevilla.

Baumol, W-J. (1993). Formal entrepreneurship theory in economics: existence and bounds. Journal of Business Venturing, 8, 197-210. https://doi.org/10.1016/0883-9026(93)90027-3

Baumol, W.-J. (2014). Stimulating growth amid recession: Entrepreneurship innovation, and the Keynesian revolution. Journal of Policy Modeling, 36, 629-635. https://doi.org/10.1016/j. jpolmod.2014.03.004

Baumol, W.-J. (2015). Joseph Schumpeter: the long run, and the short. Journal of Evolutionary Economics, 25, 37-43. https://doi.org/10.1007/s00191-013-0327-3

Berceo, G. (1978). Obras completas IV. La vida de Santo Domingo. Londres: Tamesis.

Berglund, K. (2013). Fighting against all odds: entrepreneurship education as employability training, Ephemera, 13(4), 717-735.

Bernal Guerrero, A. (2014). Competencia emprendedora e identidad personal. Una investigación exploratoria con estudiantes de Educación Secundaria Obligatoria. Revista de Educación, 363, 384-411.

Bernal Guerrero, A. (2015). Sobre la relevancia del factor personal en la investigación en educación emprendedora. En L. Núñez Cubero (Coord.) Cultura emprendedora y Educación (pp. 127-132). Sevilla: Universidad de Sevilla.

Bernal Guerrero, A. y Cárdenas, A. (2017). Evaluación del potencial emprendedor en escolares. Una investigación longitudinal. Educación XX1, 20(2), 73-94.

Bridge, S. (2017). Is «entrepreneurship» the problem in entrepreneurship education? Education + Training, 59 (7/8), 740-750. https://doi.org/10.1108/ET-02-2016-0037

Brunet, I. y Alarcón, A. (2004). Teorías sobre la figura del emprendedor. Revista de Sociología, 73, 81-103. https://doi.org/10.5565/rev/papers/v73n0.1108 
Cantillon, R. (1730). An Essay on Economic Theory. An English translation of Richard Cantillon's. Auburn: Von Mises Institute. Recuperado de: https://mises.org/library/essay-economictheory-0 (Consultado el 12/12/2018).

Cassis, Y. y Pepelasis, I. (2005). Entrepreneurship in Theory and History. Nueva York: Palgrave Macmillan. https://doi.org/10.1057/9780230522633

Castells, M. (2017). Otra economía es posible. Cultura y Economía en tiempos de crisis. Madrid: Alianza.

Chassagne, S. (1983). La formation des entrepreneurs à la période de l'industrialisation: l'example des entrepreneurs cotonniers. En AA.VV., Entreprise et Entrepreneur XIX-XXe. Siécle (pp. 290-301). Paris: Le Sorbonne.

Comisión Europea (2014). Comunicación de la Comisión al Parlamento Europeo, al Consejo, al Comité Económico y Social Europeo y al Comité de las Regiones, Balance de la Estrategia Europa 2020 para un crecimiento inteligente, sostenible e integrador. COM (2014) 130 final, del 5 de marzo de 2014. Recuperado de: http://ec.europa.eu/transparency/ regdoc/rep/1/2014/ES/1-2014-130-ES-F1-1.Pdf (Consultado el 12/12/2018).

Consejo Europeo (2000). Conclusiones de la Presidencia. Consejo Europeo de Lisboa 23 y 24 de marzo 2000. Recuperado de: https://goo.gl/pgE56j Consultado el 12/12/2018).

Coromines, J. (1981). Breve diccionario etimológico de la lengua castellana. Madrid: Gredos.

Covarrubias, S. (1674). Tesoro de la lengua castellana o española. Madrid: Imprenta de Luis Sánchez.

Cuervo, R. (1998). Diccionario de construcción y régimen de la Lengua Castellana (tomo III, letra E). Barcelona: Herder.

Diderot, M. y D'Álembert, M. (1732). Encyclopédie ou Dictionnaire raisonné des sciences, des arts et des métiers (tomo 5). Paris: Éditions sociales.

Draycott, M. y Rae, D. (2011). Enterprise education in schools and the role of competency frameworks. International Journal of Entrepreneurial Behaviour \& Research, 17(2), 127-145. https://doi.org/10.1108/13552551111114905

Du Gay, P. (2004). Against 'Enterprise' (but not against 'enterprise', for that would make no sense), Organization, 11 (1), 37-57. https://doi.org/10.1177/1350508404039777

Erkkilä, K. (2000). Entrepreneurial Education. Mapping the debates in the United States, the United Kingdom and Finland. New York: Garland Publishing.

Estienne, R. (1549). Dictionanairium latino-gallicum. Paris: Robert Estienne.

Eurydice (2016). La educación para el Emprendimiento en los centros educativos en Europa. Luxemburgo: Oficina publicaciones Unión Europea. Recuperado de: https://ec.europa. eu/epale/sites/epale/files/la_educacion_para_el_emprendimiento_en_los_centros_educativos_en_europa.pdf (Consultado el 12/12/2018).

Florea, R. y Florea, R. (2013). Entrepreneurship and Education in European Union Countries. Economy Transdisciplinary Cognition, 16(2), 75-80.

Foss, L. y Gibson, D. (2015). The entrepreneurial university: Context and institutional change. Abingdon-Oxfordshire, Reino Unido: Routledge. https://doi.org/10.4324/9781315737065

Fougère, M., Segercrantz, B. y Seeck, H. (2017). A critical Reading of the European Union's social innovation policy discourse: (Re)legitimizing neoliberalism. Organization, 24(6), 819-843. https://doi.org/10.1177/1350508416685171 
Furetière, A. (1708). Dictionnaire universel, contenant généralement tous les mots François, tant vieux que modernes \& les termes des sciences et des arts.

García-Aretio, L., Ruiz-Corbella, M. y García-Blanco, M. (2009). Claves para la educación. Actores, agentes y escenarios en la sociedad actual. Madrid: Narcea-Universidad Nacional de Educación a Distancia.

García del Dujo, A. (2011). Los límites de la Educación. Revista portuguesa de Pedagogía (extra-serie), 181-194.

García del Dujo, A. (2015). De la utilidad de las virtudes clásicas (para resguardar al sujeto de las inclemencias del tiempo). En L. Núñez Cubero (Coord.) Cultura emprendedora y Educación (pp. 61-68). Sevilla: Universidad de Sevilla.

Gibb, A. (2006). Entrepreneurship: Unique Solutions for Unique Environments. Is it possible to achieve this with the existing paradigm? Background Paper to the Plenary Presentation to the International Council for Small Business World Conference. Melbourne Australia June 18-21/2006.

Gibb, A. (2011). Concepts into practice: meeting the challenge of development of entrepreneurship educators around an innovative paradigm: The case of the International Entrepreneurship Educators Programme (IEEP). International Journal of Entrepreneurial Behaviour \& Research, 17 (2), 146-165. https://doi.org/10.1108/13552551111114914

Gill, R. (2014). If you're struggling to survive day-to-day': class optimism and contradiction in entrepreneurial discourse, Organization, 21(1), 50-67. https://doi. org/10.1177/1350508412464895

González-Domínguez F. (2004): Incidencia del marco institucional en la capacidad emprendedora de los jóvenes empresarios de Andalucía (tesis doctoral no publicada). Universidad de Sevilla. Recuperado de: https://idus.us.es/xmlui/handle/11441/14889 Consultado el 12/12/2018).

Habiby, A., Deirdre, M. y Coyle, J. (2010). El emprendedor de alta intensidad. Harvard Business Review, 88(8), 76-80.

Hannon, P. (2006). Teaching pigeons to dance: sense and meaning in entrepreneurship education. Education + Training, 48(5), 296-308. https://doi.org/10.1108/00400910610677018

Hayden, M. (2012). What Do Philosophers of Education Do? An Empirical Study of Philosophy of Education Journals. Studies in Philosophy and Education, 31, 1-27. https://doi. org/10.1007/s11217-011-9262-7

Heinonen, J. y Poikkijoki, S. (2006). An entrepreneurial-directed approach to entrepreneurship education: mission impossible? Journal of Management Development, 25(1), 80-94. https://doi.org/10.1108/02621710610637981

Hitty, U. y O'Gorman, C. (2004). What is 'enterprise education'? An analysis of the objectives and methods of enterprise education programmes in four European countries, Education + Training, 46(1), 11-23. https://doi.org/10.1108/00400910410518188

Hitty, U. (2008). Enterprise education in different cultural settings and at different school levels. En A. Fayolle y P. Kyrö (Eds.). (2008). The dynamics between entrepreneurship, environment and education (pp. 131-148) European Research in Entrepreneurship. Cheltenham-Northampton: Edward Elgar Publishing. 
Hornsby, J., Messersmith, J., Rutherford, M. y Simmons, S. (2018). Entrepreneurship everywhere: across campus, across communities and across borders. Journal of Small Business Management, 56 (1), 4-10. https://doi.org/10.1111/jsbm.12386

Hoselitz, B. (1951). The early history of entrepreneurial Theory. Explorations in Entrepreneurial History, 3(4), 193-220.

Huerta, J. (2002). Nuevos estudios de Economía Política. Madrid: Unión Editorial.

Iredale, N. (1993). Enterprise education in primary schools: a survey in two northern LEAs', Education + Training, 35(4), 22-30. https://doi.org/10.1108/00400919310041134

Johannisson, B. (2010). The agony of the Swedish school when confronted by entrepreneurship (pp 91-106). En K. Kogen y J. Sjovoll (Eds.), Creativity and Innovation. Preconditions for entrepreneurial education. Trondheim: Tapir Academic Press.

Jones, C. y Penaluna, A. (2013). Moving beyond the business plan in enterprise education. Education + Training, 55(8/9), 804-814. https://doi.org/10.1108/ET-06-2013-0077

Kenny, K. y Scriver, S. (2012). Dangerously empty? Hegemony and the construction of the Irish entrepreneur. Organization, 19(5), 615-633. https://doi.org/10.1177/1350508412448693

Knight, F. (2006). Risk, Uncertainty and Profit. New York: Dover

Komulainen, K., Naskali, P., Korhonen, M. y Keskitalo-Foley, S. (2011). Internal entrepreneurship -a Trojan horse of the neoliberal governance of education? Finnish pre-and in-service teachers' implementation of and resistance towards entrepreneurship education. Journal for Critical Education Policy Studies, 9(1), 341-374.

Lackéus, M. (2016). Value Creation as Educational Practice-Towards a new Educational Philosophy grounded in Entrepreneurship? (doctoral dissertation). Goteborg: Chalmers University of Technology. Recuperade de: http://publications.lib.chalmers.se/records/ fulltext/236812/236812.pdf (Consultado el 12/12/2018).

Lackéus, M. (2017). Does entrepreneurial education trigger more or less neoliberalism in education? Education + Training, 59(6), 635-650. https://doi.org/10.1108/ET-09-2016-0151

Lakoff, G. (1987). Women, Fire, and Dangerous Things: what categories reveal about the mind. Cambridge: Cambridge University Press. https://doi.org/10.7208/ chicago/9780226471013.001.0001

Laukkanen, M. (2000). Exploring alternative approaches in high-level entrepreneurship education: creating micro-mechanisms for endogenous regional growth. Entrepreneurship and Regional Development, 12, 25-47. https://doi.org/10.1080/089856200283072

Ley Orgánica de Educación (LOE) (Ley Orgánica 2/2006, 3 de mayo). Boletín Oficial del Estado, $n^{\circ} 106,2006,4$ de mayo.

Ley Orgánica para la Mejora de la Calidad Educativa (LOMCE) (Ley Orgánica 8/2013, 9 de diciembre). Boletín Oficial del Estado, $n^{\circ}$ 295, 2013, 10 diciembre.

Ley Orgánica de Apoyo a los Emprendedores y su Internacionalización (Ley Orgánica 14/2013, 27 de septiembre). Boletín Oficial del Estado, $n^{\circ} 233,2013,28$ de septiembre.

Lewis, K. y Massey, C (2003). Delivering enterprise education in New Zealand. Education + Training, 45(4), 197-206. https://doi.org/10.1108/00400910310478120

Marshall, A. (1961). Principles of economics. London: Macmillan.

Martínez-Rodríguez, F. y Amador, L. (2010). Educación y Desarrollo socio-económico. Contextos Educativos, 13, 83-97. 
Marulanda, F., Montoya, I. y Vélez, J. (2014). Aportes teóricos y empíricos al estudio del emprendedor. Cuadernos de Administración, 30(51), 89-99. https://doi.org/10.25100/ cdea.v30i51.46

Marttila, T. (2012). The Culture of Enterprise in Neoliberalism: Specters of Entrepreneurship. New York-London: Routledge.

Matlay, H. (2006). Entrepreneurship education: more questions than answers? Education + Training, 48(5), 293-295. https://doi.org/10.1108/et.2006.00448eaa.001

Matlay, H. (2007). Pertinent questions in entrepreneurship education: why, what, when, when and how? Education + Training, 49(8/9), 279-302.

McClure, K. (2015). Exploring curricular transformation to promove innovation and entrepreneurship: an institutional case study. Innovative Higher Education, 40(5), 429-442. https://doi.org/10.1007/s10755-015-9325-8

Menger, C. (1997). Principios de Economía Política. Madrid: Unión Editorial.

Mill, J. S. (1984). El Utilitarismo. Madrid: Alianza.

Moberg, K. (2014). Two approaches to entrepreneurship education: The different effects of education for and through entrepreneurship at the lower secondary level. The International Journal of Management Education 12(3), 512-528. https://doi.org/10.1016/j. ijme.2014.05.002

Mwasalwiba, S. (2010). Entrepreneurship education: a review of its objectives, teaching methods, and impact indicators, Education + Training, 52(1), 20-47. https://doi. org/10.1108/00400911011017663

Nabi, G. y Liñán, F. (2011). Graduate Entrepreneurship in Developing Countries: Intentions, Education and Development. Education + Training, 53(5), 325-334. https://doi. org/10.1108/00400911111147668

Nabi, G., Liñán, F., Fayolle, A., Krueger, N. y Walmsley, A. (2017). The impact of entrepreneurship education in higher education: A systematic review and research agenda. Academy of Management Learning and Education, 16(2), 277-299. https://doi.org/10.5465/ amle.2015.0026

Neck, H. y Greene, P. (2011). Entrepreneurship Education: Known Worlds and New Frontiers. Journal of Small Business Management, 49(1), 55-70. https://doi.org/10.1111/j.1540627X.2010.00314.X

Nicot, J. (1606). Thresor de la Langue Francoyse Tant Ancienne que Moderne. Paris: Duvacer. Recuperado de: https://gallica.bnf.fr/ark:/12148/bpt6k50808z.image (Consultado el 12/12/2018).

Nueno, P. (2001). Emprendiendo. El arte de crear empresas y sus artistas. Bilbao: Deusto.

Núñez Cubero, L. (2015) (Coord.). Cultura Emprendedora y Educación. Sevilla: Universidad de Sevilla.

O'Rourke, B. (2014). Learning from interacting: language, economics and the entrepreneur. On the Horizon, 22(4), 245-255. https://doi.org/10.1108/OTH-01-2014-0002

Packham, G., Jones, P., Miller, C., Pickernell, D. y Thomas, B. (2010), Attitudes towards entrepreneurship education: a comparative analysis, Education + Training, 52 (8/9), 568-586. https://doi.org/10.1108/00400911011088926

Pallet, J. (1604). Diccionario muy copioso de la lengua española y francesa. París: Guillemot. 
Peña-Calvo, J., Cárdenas, A., Rodríguez-Martín, A y Sánchez-Lissen, E. (2015). La cultura emprendedora como objetivo educativo: marco general y estado de la cuestión. En L. Núñez Cubero (Coord.) Cultura emprendedora y Educación (pp. 19-60). Sevilla: Universidad de Sevilla.

Pepin, M. (2012). Enterprise education: a Deweyan perspective, Education + Training, 54(8/9), 801 - 812. https://doi.org/10.1108/00400911211274891

Pérez-Sánchez B. (2011). Reseña crítica del empresario en la fisiocracia y la economía clásica. Hitos de Ciencias Económico Administrativas 17(47), 27-38.

Pfeilstetter, R. (2011). El emprendedor. Una reflexión crítica sobre usos y significados actuales de un concepto. Gazeta de Antropología, 27(1), 1-11.

Pittaway, L. y Cope, J. (2007). Entrepreneurship Education. A systematic review of evidence. Internacional Small Business Journal, 25(5), 479-510. https://doi.org/10.1177/0266242607080656

Polo, L. (1990). Hacia un mundo más humano. Cuadernos de Empresa y Humanismo, 32.

Ray, A. (1994). Dictionnaire historique de la langue française. París: Le Robert.

Real Academia Española (1732). Diccionario de Autoridades, tomo IIII.

Real Academia de la Lengua Española (1780) Diccionario de la Lengua Española compuesto por la Real academia española, reducido á un tomo para su más fácil uso. (1 $1^{a}$ edición). Madrid: Viuda de J. Ibarra, impresora de la Real Academia.

Real Academia de la Lengua Española (1791) Diccionario de la Lengua Española compuesto por la Real academia española, reducido á un tomo para su más fácil uso. (3ª edición). Madrid: Viuda de J. Ibarra, impresora de la Real Academia.

Real Academia de la Lengua Española (1925) Diccionario de la Lengua Española. (15ª edición). Real Academia de la Lengua Española (2001) Diccionario de la lengua española. (22 edición). Real Academia de la Lengua Española (2014) Diccionario de la lengua española. (23a edición). Richelet, P. (1680). Dictionnaire Français des Mots et des Choses. Geneve: Widerhold. Recuperado de: https://gallica.bnf.fr/ark:/12148/bpt6k509323/f1.image (Consultado el 12/12/2018)

Rodríguez-Sedano, A. (1999). Una teoría del emprendedor: Ensayo de genealogía conceptual (tesis doctoral). Barcelona: IESE.

Rodríguez-Sedano, A. y López de Pedro, J. (2013). Pasado, presente y futuro del emprendedor. La racionalidad en la actividad emprendedora. Recuperado de: http://www.espanito. $\mathrm{com} /$ pasado-presente-y-futuro-del-emprendedor-la-racionalidad-en-la.html (Consultado el 12/12/2018).

Rosendahl, L., Sloof, R. y Van Praag, M. (2014). The Effect of Early Entrepreneurship Education: Evidence from a Field Experiment. European Economic Review, 72, 76-97. https:// doi.org/10.1016/j.euroecorev.2014.09.002

Shane, S. y Venkataraman, S. (2000). The promise of entrepreneurship as a field of research. Academy of Management Review, 25, 217-226. https://doi.org/10.5465/amr.2000.2791611

Savary des Brûlons (1748). Dictionnaire universel du commerce, tomo II.

Say, J.-B. (1821). Tratado de economía política o exposición sencilla del modo con que se forman, se distribuyen y se consumen las riquezas. Madrid: Imprenta Villalpando

Seamus, N. (2013). Richard Cantillon. The father of economics, Ireland History, 21(2), 20-23.

Segura, A. (1994). La inserción de la Persona en la Empresa. Cuadernos Empresa Humanismo, 41. 
Schumpeter J. (1966). Capitalismo, socialismo y democracia. Madrid-México-Buenos Aires: Aguilar.

Thierry, J. (1564). Dictionnaire Françoise-Latin. Paris: Dupy-Macé.

Tobón, S. (2001). Aprender a emprender: un enfoque curricular. Medellín: Fundación Universitaria del Oriente.

Toca, C. (2010). Consideraciones para la formación en emprendimiento: explorando nuevos ámbitos y posibilidades. Estudios Gerenciales, 26(117), 41-60. https://doi.org/10.1016/ S0123-5923(10)70133-9

Toro, J. y Ortegón, A. (1999). Corriendo el telón del concepto emprendedor. Revista Escuela de Administración de Negocios, 37, 133-141.

Van Praag, M. (1999). Some classic views on entrepreneurship. De Economist, 147, 311-335. https://doi.org/10.1023/A:1003749128457

Veciana, J. (1996). Emprendedor o empresario. Innovando. Boletín informativo del ICESI, 17, 2-3.

Veciana, J. (2005). La creación de empresas. Un enfoque gerencial. Colección Estudios Económicos, 33. Barcelona: La Caixa.

Verin, H. (2011). Entrepreneurs, enterprise, histoire d'une idée. Paris: Garnier.

Vesper, K. H., Gartner, W. B. (1997). Measuring progress in entrepreneurship education. Journal Business Venturing, 12(5), 403-421. https://doi.org/10.1016/S0883-9026(97)00009-8

Villamor, P. y Prieto, M. (2014). El espíritu emprendedor en educación: un análisis pedagógico. Procedia. Social and Behavioral Sciences, 139, 153-159.

Volkmann, C., Wilson, K., Mariotti, S., Rabuzzi, D., Vyakarnam, S. y Sepulveda, A. (2009). Educating the next wave of entrepreneurs-unlocking entrepreneurial capabilities to meet the global challenges of the 21st Century. A Report of the Global Education Initiative. Ginebra: Word Economic Forum.

Weber, M. (1995). La ética protestante y el espiritu del capitalismo. Barcelona: Península.

Westhead, P. y Solesvik, M. (2015). Entrepreneurship education and entrepreneurial intention: Do female students benefit? International Small Business Journal, 34(8), 979-1003. https://doi.org/10.1177/0266242615612534

Zaratiegui, J. (2002). Alfred Marshall y la teoría económica del empresario. Valladolid: Universidad de Valladolid. 